\title{
GmFULa improves soybean yield by enhancing carbon assimilation without altering flowering time or maturity
}

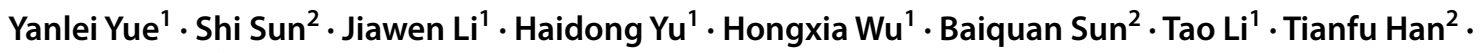 \\ Bingjun Jiang ${ }^{2}$ (I)
}

Received: 30 April 2021 / Accepted: 4 July 2021 / Published online: 16 July 2021

(c) The Author(s) 2021

\begin{abstract}
Key message GmFULa improved soybean yield by enhancing carbon assimilation. Meanwhile, different from known yield-related genes, it did not alter flowering time or maturity.

Abstract Soybean (Glycine max (L.) Merr.) is highly demanded by a continuously growing human population. However, increasing soybean yield is a major challenge. FRUITFULL (FUL), a MADS-box transcription factor, plays important roles in multiple developmental processes, especially fruit and pod development, which are crucial for soybean yield formation. However, the functions of its homologs in soybean are not clear. Here, through haplotype analysis, we found that one haplotype of the soybean homolog GmFULa (GmFULa-HO2) is dominant in cultivated soybeans, suggesting that GmFULa-HO2 was highly selected during domestication and varietal improvement of soybean. Interestingly, transgenic overexpression of GmFULa enhanced vegetative growth with more biomass accumulated and ultimately increased the yield but without affecting the plant height or changing the flowering time and maturity, indicating that it enhances the efficiency of dry matter accumulation. It also promoted the yield factors like branch number, pod number and 100-seed weight, which ultimately increased the yield. It increased the palisade tissue cell number and the chlorophyll content to promote photosynthesis and increase the soluble sugar content in leaves and fresh seeds. Furthermore, GmFULa were found to be sublocalized in the nucleus and positively regulate sucrose synthases ( $S U S S$ ) and sucrose transporters (SUTS) by binding with the conserved CArG boxes in their promoters. Overall, these results showed GmFULa promotes the capacity of assimilation and the transport of the resultant assimilates to increase yield, and provided insights into the link between GmFULa and sucrose synthesis with transport-related molecular pathways that control seed yield.
\end{abstract}

Keywords Soybean (Glycine max (L.) Merr.) · GmFULa $\cdot$ Yield $\cdot$ Biomass $\cdot$ Palisade tissue $\cdot$ Sucrose synthesis $\cdot$ Transport

Abbreviations
CDS Coding sequence
$D A G \quad$ Day after germination

Communicated by Prakash P. Kumar.

Tao Li

litao0504@henau.edu.cn

Tianfu Han

hantianfu@caas.cn

Bingjun Jiang

jiangbingjun@caas.cn

1 College of Life Sciences, Henan Agricultural University, Zhengzhou 450002, China

2 MARA Key Lab of Soybean Biology (Beijing), Institute of Crop Sciences, The Chinese Academy of Agricultural Sciences, Beijing 100081, China
FUL FRUITFULL

SUS Sucrose synthase

SUT Sucrose transporter

ZGDD Zigongdongdou

\section{Introduction}

Soybean (Glycine max (L.) Merr.) provides large amounts of edible oils and vegetable proteins for humans and livestock, and thus, demand for soybean is increasing globally due to human population growth. However, available cultivated land resources are largely limited, meaning that increasing soybean unit yield, as a way to increase the total yield, is a major challenge. Soybean yield is based on pod number per plant, seed number per pod and 100-seed weight (Van Roekel et al. 2015; Yan et al. 2017; Bianchi et al. 2020). 
Taking into account that soybean is sensitive to photoperiod, maturity loci have important roles in yield. However, there are many yet unknown quantitative loci reported to be linked to yield-related traits in SoyBase (https://www.soyba se.org/) but only a few loci have been molecularly identified. Among them, GmCYP78A10 is related to pod number and 100 -seed weight and $L n$ is a major gene controlling fourseed pod development in soybean (Jeong et al. 2012; Wang et al. 2015; Sayama et al. 2017). GsCID1 is responsible for 100-seed weight in wild soybean (Hu et al. 2020). Recently, GmKIX8-1 was demonstrated to be associated with soybean seed weight (Nguyen et al. 2021). However, the molecular mechanism controlling yield in soybean is largely unknown.

FRUITFULL (FUL), a MADS-box transcription factor, has essential and pleiotropic roles in multiple developmental processes including shoot initiation, reproductive transition, inflorescence differentiation and fruit development (Ferrandiz et al. 2000; Balanza et al. 2019; Maheepala et al. 2019; Zhang et al. 2019a; Zhao et al. 2019). In Arabidopsis, FUL (AGL8) is the main member of the regulatory network that determines fruit growth pattern (Mandel and Yanofsky 1995; Alvarez-Buylla et al. 2019). FUL down-regulates APETALA2 and INDEHISCENT, and they two promote pod elongation (Mandel and Yanofsky 1995; Balanza et al. 2019; Di Marzo et al. 2020). FUL is necessary for terminal flower formation in seedless states (Balanza et al. 2019). FUL also contributes to the differentiation of inflorescence, stem, leaf and carpel (Zhang et al. 2013; Yao et al. 2019). Similar functions were found in the pea and cotton homologous genes VEGETATIVE1 and GhMADS22, respectively (Berbel et al. 2012; Zhang et al. 2013). CsFUL1 regulates fruit length in cucumber, and DEFH28, a homologous gene in Antirrhinum japonicum, regulates carpel wall differentiation and fruit ripening (Müller et al. 2001; Zhao et al. 2019). In tomato, TDR4/FUL1 and MBP7/FUL2 are involved in cell wall modification and affect fruit ripening without dependence on ethylene (Bemer et al. 2012; Li et al. 2019). FUL can also modulate plant architecture. Rice homolog OsMADS18 is negatively related to the number of tillers, and controls the branch angle by inhibiting SAUR10 (SMALL AUXIN UPREGULATED RNA 10) expression (Bemer et al. 2017).

In addition, FUL functions in the formation of secondary metabolites. VmTDR4 is involved in the accumulation of anthocyanins during the normal ripening period in blueberries, and DR4/FUL1 and MBP7/FUL2 participate in the synthesis of volatile substances in tomato fruits (Jaakola et al. 2010; Bemer et al. 2012) and affect the accumulation of pigment in tomato maturity (Wang et al. 2014). Moreover, in Arabidopsis, FUL can intertalk with hormone- and light-signaling pathways. It directly regulates cytokinin oxidase genes $C K X 5$ and $C K X 6$, plant pigment interaction factor PIL1, della family genes $R G L 2$ and GAI, and auxinresponsive gene SAUR10 (Bemer et al. 2017; Di Marzo et al.
2020). FUL protein can bind to the CArG box in the promoter of the SHP gene to regulate the development of fruit petal and embryo and limit pod dehiscence of Arabidopsis thaliana by regulating cell proliferation (Sehra and Franks 2017). In soybean, Jia et al. (2015) found eight $A P 1 / F U L$ like genes: GmFULa (Glyma.06G205800); GmFULb (Glyma.04G159300); GmFULc (Glyma.05G018800); GmFULd (Glyma.17G081200); GmAPla (Glyma.16G091300); GmAP1b (Glyma.08G269800); GmAPlc (Glyma.01G064200); and GmAPld (Glyma.02G121600). Few studies have been performed on these soybean $F U L$ homologs, except that GmFULa was found to be highly expressed in the root and shoot apices and might be involved in plant architecture and yield. Particularly, the function of GmFULa and its mode of action are unclear.

Here, we analyzed the molecular function of GmFULa. First, we found that it has six haplotypes with GmFULa-HOI and GmFULa-HO2 dominant. GmFULa-HO1 is dominant in wild soybeans, while GmFULa-HO2 is dominant in cultivated soybeans especially southern cultivars, indicating that it is an elite allele for soybean breeding. Overexpression of GmFULa-HO2 results in more biomass and higher yield with increased seed number and weight. We further show that whole-soluble sugar contents were increased in fresh leaves and seeds. Consistently, GmFULa bound to the promoters of GmSUS12 and GmSUT5 and activated their expression. The cell distribution in palisade tissue regulated by GmFUL $a$ and chlorophyll content increased significantly with high photosynthetic efficiency in an overexpression line. Our results show that the GmFULa-GmSUSs/GmSUTs pathway regulates the seed yield of soybean, controls the cell number of palisade tissue, and enhances organic matter accumulation by increasing whole-soluble sugar contents in leaf tissue. In contrast, it does not affect flowering time or maturity.

\section{Materials and methods}

\section{Cloning and sequence analysis of GmFULa}

The sequences of GmFUL family genes and their encoding proteins were obtained from Phytozome (https://phyto zome.jgi.doe.gov). Conserved domains were searched in the NCBI database. The CDS of GmFULa was amplified from the soybean cultivar Zigongdongdou (ZGDD) with the primers listed in Supplementary Table S1. Then, the PCR products were cloned into the pZeroBack/blunt vector (TianGen, Beijing, China). Sanger sequencing was then performed to confirm the sequences and variations (Shanghai Sangon Biological Engineering Technology and Service CO., LTD, Zhengzhou, China). 


\section{Haplotype analysis of GmFULa}

Publicly available genome resequencing data (NCBI: SRP062560, SRP045129) were used. These sequences were mapped to the Williams 82 genome (v275) using bwa v0.7.10 with default parameters. SNPs/indels were called using the UnifiedGenotyper module (-stand_call_conf 30.0 -stand_emit_conf 10.0) of the GenomeAnalysisTK suite (https://gatk.broadinstitute.org/) (Zhang et al. 2019b). The polymorphism information of GmFULa was further extracted. The SNP/InDel sites located in the CDS region and including missense mutations were selected to define haplotypes and perform haplotype analysis (Jiang et al. 2019).

\section{Plant materials and growth conditions}

ZGDD is a photoperiod-sensitive cultivar suitable of low latitude conditions. In this study, ZGDD was used as a wildtype control for molecular analysis including genetic transformation. To investigate soluble sugar content, chlorophyll content, sucrose synthase activity, biomass, cell morphology and gene expression, plants were grown in pots containing a 1:1 mixture of forest vermiculite in a light chamber (20000 Lux) under short-day condition $(12 \mathrm{~h} / 12 \mathrm{~h})$ at $25^{\circ} \mathrm{C}$ with $60 \%$ humidity.

Plants for the investigation of maturity, yield-related traits and photosynthetic activity were grown under field conditions (with $1.5 \mathrm{~m}$ row length, $75 \mathrm{~cm}$ row spacing, $10 \mathrm{~cm}$ plant spacing, three replicates and completely randomized design) from November of 2016, 2019 and 2020 to next Aprils in tropical city of Sanya $\left(18.1^{\circ} \mathrm{N}, 109.2^{\circ} \mathrm{E}\right.$, mean temperature $\left.26^{\circ} \mathrm{C}\right)$, Hainan province, China.

\section{Creation of transgenic overexpression plants of GmFULa}

For the GmFULa overexpression construct, the full-length coding sequence of GmFULa from ZGDD was cloned into the binary vector pTF101.1 between the XbaI and SacI sites, downstream of the constitutive Cauliflower Mosaic Virus 35S promoter (Yue et al. 2017). The transformation of soybean followed the method of affecting Agrobacteriummediated using the cotyledonary node explant (Paz et al. 2004). Transgenic plants were verified by PCR-based markers with primers listed in Supplementary Table S1. The soybean transgenic lines were advanced to the $\mathrm{T} 5$ generation.

\section{Soluble sugar determination}

The middle leaflet of the second fully expanded trifoliolate leaves in the V2 stage and the seed of R6 stage were used. The relative content of soluble sugar was determined with the Anthrone-sulfuric acid colorimetry method using the soluble sugar extract of ZGDD as reference (Huang et al. 2020).

\section{Sucrose synthase activity measurement}

The sucrose synthase activity was measured with a Sucrose Synthase Activity Assay Kit (boxbio, Beijing, China). The relative sucrose synthase activity was expressed as the ratio between the sucrose synthase activities of transgenic lines and ZGDD.

\section{Chlorophyll concentration measurement}

The middle leaflet of the second fully expanded trifoliolate leaves in the R2 stage (full blooming) were collected. The chlorophyll was extracted and measured with the method described by Xu et al. (2013).

\section{Biomass measurement}

The whole plants of 3, 7 and 15 DAG were used and cut into separate shoot and root parts from the cotyledon node. Then, the shoot and root samples were sterilized at $105{ }^{\circ} \mathrm{C}$ for $30 \mathrm{~min}$, dried to constant weight, and weighed.

\section{Photosynthesis rate analysis}

For each group, fifteen plants were randomly selected. The middle leaflet of the second fully expanded trifoliolate leaves in the V3 stage was used. The photosynthesis measurement was conducted with the Li-6400 portable photosynthesis measuring system (LI-COR, USA) (Singsaas et al. 2004; Xu et al. 2013; Busch 2018). The photo-synthetically active radiation was set up as $1,200 \mu \mathrm{mol}$ photons $\mathrm{m}^{-2} \mathrm{~s}^{-1}$ (Xu et al. 2013).

\section{Leaf morphology and anatomy}

The middle leaflet of the third trifoliolate leaf from top was sampled for anatomy analysis, which was performed by Servicebio, China (https://www.servicebio.cn/). The sections were stained with safranin $\mathrm{O}$ and fast green (Langdale et al. 1989), mounted with neutral balsam and scanned with a Pannoramic 250 Flash II Scanner (3DHISTECH Kft., Budapest, Hungary). The thicknesses of the leaf, spongy mesophyll, stratum corneum and palisade, the numbers of veins and palisade tissue cells, and the cell sizes of the upper epidermis and palisade cells were evaluated at five points. 


\section{Gene expression analysis}

The middle leaflet of the second expansion trifoliolate leaves of soybean V3 stage was sampled. RNA of transgenic plants and wild-type ZGDD was isolated using TRIZol (ET111), and reverse transcribed into cDNA with Easyscript ${ }^{\circledR}$ onestep gDNA removal and cDNA synthesis superMix (AT311) (Transgen Biotech, Beijing, China). The cDNA concentrations were normalized to the GmActin expression levels for quantitative PCR analysis (Quant Studio ${ }^{\mathrm{TM}} 12 \mathrm{~K}$ Flex). The qPCR primers are listed in Supplementary Table S1. The primer specificity and efficiency verification used primer blast in NCBI (https://www.ncbi.nlm.nih.gov/). PCR cycle conditions as hold stage: $95{ }^{\circ} \mathrm{C} 20 \mathrm{~s}$, then PCR stage: $\left(95^{\circ} \mathrm{C} 1 \mathrm{~s}\right.$, $\left.60{ }^{\circ} \mathrm{C} 20 \mathrm{~s}\right) \times 50$ cycle, and melt curve stage: $95^{\circ} \mathrm{C} 15 \mathrm{~s}$, $60{ }^{\circ} \mathrm{C} 1 \mathrm{~min}, 95^{\circ} \mathrm{C} 15 \mathrm{~s}$. The relative expression levels were estimated using the $2^{-\Delta \Delta C T}$ method (Taylor et al. 2019). Three biological replicates were included.

\section{Transient expression in soybean protoplasts}

The GmFULa CDS without the stop codon was amplified and fused to the 5' end of the open reading frame encoding GFP in pTF101 (Yue et al. 2017), which was driven by the CaMV35S promoter. For the ProGmSUS12YFP construct, a genomic DNA sequence (from -1224 to $-1 \mathrm{bp}$ ) upstream of the GmSUS12 coding sequence was amplified using sequencespecific primers and the sequence was cloned into KpnI and XhoI sites of the pYFPLT vector, which contains the yellow fluorescent protein (YFP) coding sequences. The recombinant construct was transformed into soybean protoplasts.

Selected 14-day soybean leaves were cut into $1 \mathrm{~mm}$ strips and incubated with the enzyme digestion solution (1\% Cellulase "Onozuka" Rs, 0.5\% Pectolase Y-23, 9\% Mannitol) in the dark for $5 \mathrm{~h}$ at $25^{\circ} \mathrm{C}$. An equal volume of CPW9M solution was added (Frearson et al. 1973). Digested tissues were filtered through a molecular sieve with 100 mesh number. After centrifugation at $200 \mathrm{~g}$ for $5 \mathrm{~min}$ at $4{ }^{\circ} \mathrm{C}$, the protoplast was washed and precipitated three times with CPW9M. Plasmid $(10 \mu \mathrm{g})$ was added to $100 \mu \mathrm{L}$ protoplasts, which were resuspended in MMG and incubated with $110 \mu \mathrm{L}$ PEG4000 solution for $15 \mathrm{~min}$ at $25{ }^{\circ} \mathrm{C}$. After that, $600 \mu \mathrm{L}$ W5 stop solution was used to end the transfection the protoplasts were washed twice with CPW9M or W5 as described previously (Yoo et al. 2007). Finally, protoplasts were cultured in $\mathrm{CPW} 9 \mathrm{M}$ for $20 \mathrm{~h}$ in the dark at $25^{\circ} \mathrm{C}$. Fluorescence images were taken using a confocal laser scanning microscope.

\section{Protein expression, purification and electrophoretic mobility shift assay (EMSA)}

For expression of GmFULa protein in bacteria, the GmFULa full-length coding sequences were inserted into the inducible expression vector pET-32a (with $6 \times$ His Tag) between BamHI and SacI sites. The resulting plasmids were transformed into Escherichia coli strain Rosetta (DE3) and induced using $0.4 \mathrm{mM}$ isopropyl-b-ß-1-thiogalactopyranoside (IPTG) at $25{ }^{\circ} \mathrm{C}$ for $12 \mathrm{~h}$. The recombinant protein was purified using Proteinlso ${ }^{\mathrm{R}} \mathrm{Ni}-\mathrm{NTA}$ Resin (Transgen biotech, Beijing, China) according to the manufacturer's protocol. For GmSUSs and GmSUTs, the probe fragment consisted of a region of $40 \mathrm{bp}$ with the canonical CArG box $\left(\mathrm{C}[\mathrm{A} / \mathrm{T}]_{8} \mathrm{G}\right)$ in the center (Table $\mathrm{S} 1$ for primer sequences). The mutated $\mathrm{CArG}$ box fragment TM was CCGCG (AATAT) in the mid region of the TG motif. The probes were labeled using the EMSA Probe Biotin Labeling Kit (Beyotime Biotechnology). The same fragments without biotin labeling were used as competitors. The protein-DNA complexes were separated with $6 \%$ native polyacrylamide gels. The Biotin-labeled probes were visualized using Chemiluminescent Biotin-labeled Nucleic Acid Detection Kit (Beyotime Biotechnology) according to the manufacturer's protocol.

\section{Results}

\section{GmFULa has two highly conserved, dominant haplotypes distributed in both wild and cultivated soybeans}

Based on the public soybean resequencing data (NCBI: SRP020131, SRP062560, SRP045129 and PRJNA589345) from several whole-genome resequencing studies (Lam et al. 2010; Zhou et al. 2015; Zhang et al. 2019b), we analyzed the polymorphisms of GmFULa in 549 lines including 86 wild soybeans. We found 161 variation sites, of which there were 141 SNPs (single nucleotide polymorphisms) and 20 indels (insertions and deletions), including two synonymous mutation sites (black lines in Fig. 1a) and three missense mutation sites in the CDS (coding sequence) region (blue lines in Fig. 1a). Based on these five CDS variations, GmFULa was divided into six haplotypes: H01-H06, of which H01 and H02 were the most dominant (Fig. 1b). Moreover, in wild soybean, H01 was nearly the only haplotype. However, H01 and $\mathrm{H} 02$ were distributed nearly equally in the 80 widely planted cultivated soybeans from Northeast China, while H02 was dominant in the 54 widely planted cultivated soybeans from the Huang-Huai-Hai region and Southern China (Fig. 1b). These results indicated that $\mathrm{H} 02$ is an elite haplotype related to soybean geographical adaptation. Combined with the observation that GmFULa was highly expressed in the shoot apices (Jia et al. 2015), which strongly indicates that GmFULa should have an important role in yield. However, its actual function is unclear. 


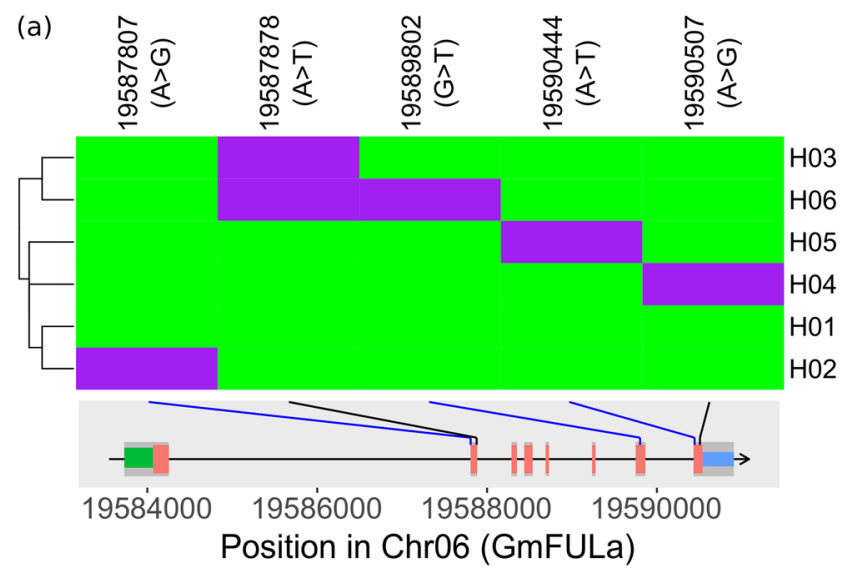

(b)
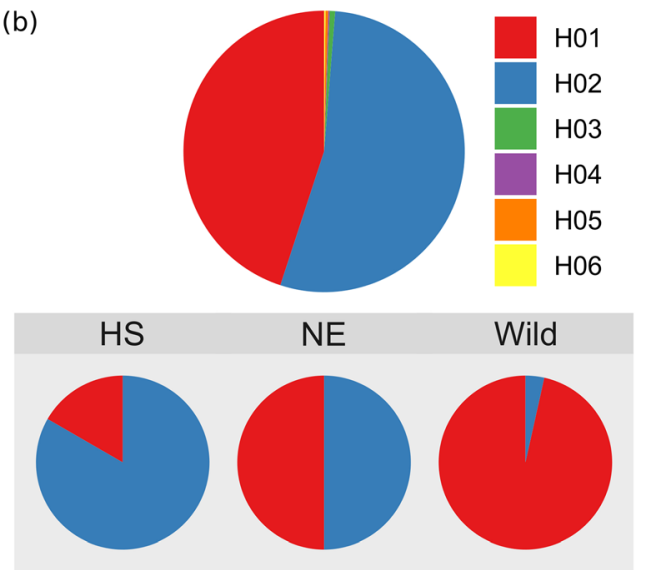

(c)
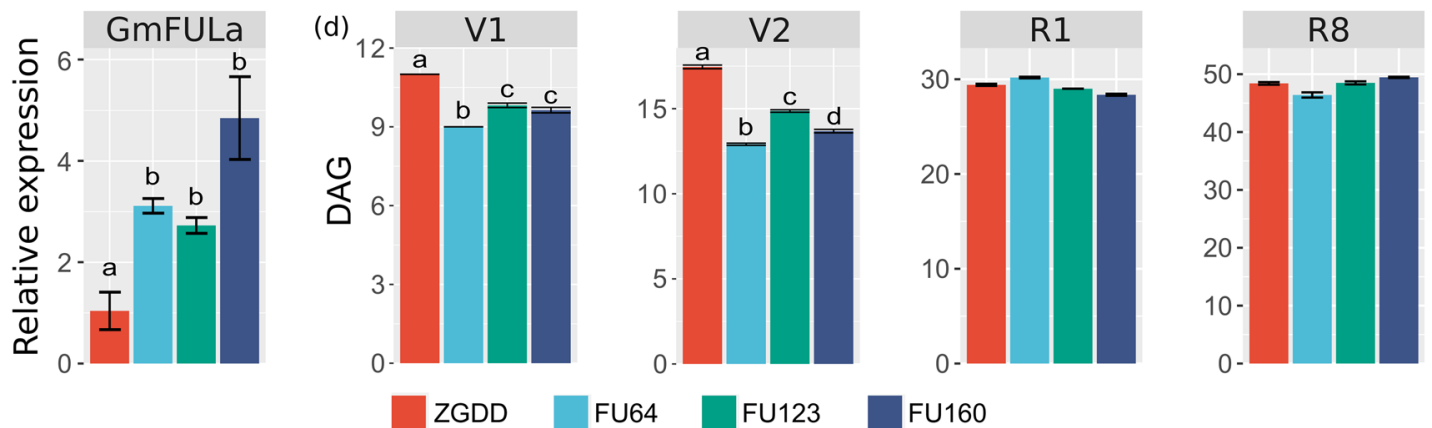

Fig. 1 Polymorphisms and haplotypes of GmFULa and the effect of one dominant haplotype on maturity. a Polymorphisms and haplotypes of GmFULa. Bottom inset shows the exon-intron structure of GmFULa and the location of polymorphisms, where green, red and blue bars indicate 5' UTR, CDS and 3' UTR, respectively, and blue and black lines, respectively, indicate missense and synonymous variants. The upper panel shows the haplotypes of GmFULa. Reference alleles are in green; alternative alleles are in purple. b Distribution of GmFULa haplotypes. The upper panel shows the general distribution of GmFULa haplotypes detected in all soybeans. The bottom panel shows the distribution of $\mathrm{H} 01$ and $\mathrm{H} 02$ haplotypes in cultivars from Northeast China (NE), cultivars from the Huang-Huai-Hai valley region and South China (HS), and wild soybeans (Wild). c Verification of GmFULa overexpression in transgenic lines by real-time

\section{GmFULa promotes soybean vegetative growth without affecting maturity}

As GmFULa has three highly conserved homologs in soybean genome, and especially GmFULa is nearly identical to GmFULb with the identity rate as $92.2 \%$ in amino acid sequence and $94.1 \%$ in nucleotide sequence, respectively (Supplementary Figure S1), it is reasonable that GmFULa nullification through CRISPR/Cas9-based gene editing system will be compensated by these homologs especially GmFULb. Thus, to further evaluate the function of GmFULa, we only conducted a conventional overexpression analysis at this time. We cloned GmFULa from soybean variety Zigongdongdou (ZGDD, with the haplotype of GmFULa-HO2) and constructed several transgenic

quantitative PCR analysis. GmActin was used as an internal control. Values are given as mean $\pm S E$ of three biological replicates with letters showing if there is a significant difference between groups (OneWay ANOVA; Tukey HSD test at, <0.05). d The growth stages of transgenic soybean lines and control soybean ZGDD. V1, V2, R1 and R8 are soybean growth stages of one unrolled trifoliolate leaf, two unrolled trifoliolate leaves, beginning flowering and full maturity, respectively. ZGDD: control soybean Zigongdongdou (transgenic receptor). FU64, FU123 and FU160 are independent transgenic lines overexpressing GmFULa. The data represent the mean $\pm \mathrm{SE}$ of $\geq 20$ biological replicates with letters showing if there is a significant difference between groups (One-Way ANOVA; Tukey HSD test at, $<0.05)$

overexpression lines where GmFULa was driven by the CaMV35S promoter. Homozygous transgenic lines were screened and identified by the combination of an herbicide test and PCR identification each generation (Supplementary Figure S2a and b). As expected, the expression levels of GmFULa, examined by quantitative PCR (qPCR), were significantly higher in the transgenic plants (FU64 and FU123, $p<0.01$; FU160, $p<0.05$ ) than in the control ZGDD (Fig. 1c). However, when determining whether GmFULa promotes plant growth, we found that although the transgenic and control plants reached the vegetative stages VE (emergence) and VC (unifoliolate leaves unrolled) on the same day, days to the subsequent vegetative stages V1 (one trifoliolate leaf unrolled) and V2 (two trifoliolate leaves unrolled) of transgenic lines were, respectively, about 2 days 
and 5 days shorter than those of the control $(p<0.01)$. In contrast, the days to the reproductive stages R1 (first flowering) and R8 (full maturity) of transgenic lines were nearly equal to those of the control (Fig. 1d). These results indicated that GmFULa neither affected germination nor maturity but promoted vegetative growth.

\section{GmFULa enhances the accumulation of biomass in a robust way}

Consistent with our previous observation, in terms of whole plants, transgenic lines had the same number of nodes and leaves as the control ZGDD at 3 and 7 DAG (days after germination), while they had one more node and trifoliolate leaves at 15 DAG (Fig. 2a-c). Wild-type plants reached the vegetative stage of V1 at 15 DAG, while transgenic lines had already entered the next stage of V2 (Fig. 2c), indicating that transgenic lines are more vigorous than the wild-type soybean. Moreover, for the three observation time points (3, 7 , and $15 \mathrm{DAG}$ ), vegetative organs (cotyledons and leaves) were significantly bigger in transgenic soybeans than the wild-type control (Fig. 2d-f). Consistently, transgenic lines accumulated significantly higher dry biomass of both shoot and root compared to wild-type plants at 3,7 and 15 DAG (Fig. 2g). These results suggested that GmFULa promotes vegetative biomass accumulation in soybean by increasing the vigor of plants.

\section{GmFULa regulates soybean sink content with increasing soybean yield}

Although GmFULa promotes vegetative growth and biomass accumulation leading to increase the source capacity at the vegetative stage, it is necessary to further confirm whether GmFULa can promote the transformation of carbohydrate source-sink to increase soybean yield. To correspond with actual production practices, we evaluated the yield potentiality of GmFULa under natural field conditions. Transgenic overexpression lines (FU64, FU123, FU160) and wildtype control (the transgenic receptor cultivar ZGDD) were grown in an experimental field in Sanya, Hainan province. Yield-related traits were investigated, including the branch number, plant height, node number, pod number, and seed number as well as overall yield. In keeping with the results of the experiments performed in incubators, the GmFULaoverexpression plants grew better compared to the wild-type control with more pods and more branches but similar height (Fig. 3). The seed sizes of transgenic lines were also bigger than the wild type (Fig. 3a). Consistently, the yield-related traits of branch number, pod number, and seed number as well as overall yield all increased significantly $(p<0.01)$ in all transgenic lines and the node number also increased in two transgenic lines (Fig. 3b-g). However, plant height did not show a significant difference between transgenic lines and wild-type control (Fig. 3c). Moreover, FU160 had the highest GmFULa expression level among the three overexpression lines; similarly, it had the highest values of yieldrelated traits branch number, node number, pod number, and seed number and overall yield. These results indicated that GmFULa increased soybean source content and promoted the source-sink transformation to increase soybean yield. No significant difference was observed in plant height between the overexpression lines and the wild-type control (Fig. 3c), suggesting that GmFULa is a candidate gene for ideal plant architecture with shorter node spacing that is more conducive to the utilization, transportation and storage of energy and materials from the source organ.

\section{GmFULa modifies the cell distribution of source organ leaf}

To understand how GmFULa regulates yield-related traits, we further investigated how leaves, a major source organ, were changed by GmFULa overexpression. Various experiments provided direct hints that GmFULa increases leaf size and thickness, so the potential role of GmFULa in the shape and morphology of leaf cells should be clarified. We performed microscopic observations of leaf transects of a middle leaflet at the same trifoliolate node 25 DAE (days after emergence) of the typical overexpression line FU160 and the wild-type control ZGDD (Fig. 4a, b). Consistently, the leaf of the transgenic line was significantly thicker than that of the wild-type control (Fig. 4c-e). The numbers of veins and palisade tissue cells in transgenic lines increased significantly compared to those of the wild-type control (Supplementary Table S2 and Fig. 4d). The spongy mesophyll, stratum corneum and palisade were thicker in the transgenic line than in the wild-type control (Fig. $4 \mathrm{f}-\mathrm{h}$ ), though the difference was not significant for spongy mesophyll. In addition, the cell size of the upper epidermis (FU160 $=434 \mu \mathrm{m}^{2}$, $\mathrm{ZG}=272 \mu \mathrm{m}^{2}$ ) increased significantly in transgenic line FU160 while the cell size of the lower epidermis decreased (Fig. 4i, j and Supplementary Table S2). Palisade cell size in FU160 was not significantly different from the one in control ZGDD (Fig. 4k). These data suggest that GmFULa has an important role in regulating cell distribution and palisade development in leaf photosynthesis.

\section{GmFULa regulates source-sink balance with carbon assimilation and transfer}

To study the physiological function of GmFULa in soybean growth, we determined chlorophyll content, photosynthesis rate, soluble sugar content and sucrose synthase activity. For chlorophyll content, leaf blades were sampled with a hole punch from the middle leaflet of the second 

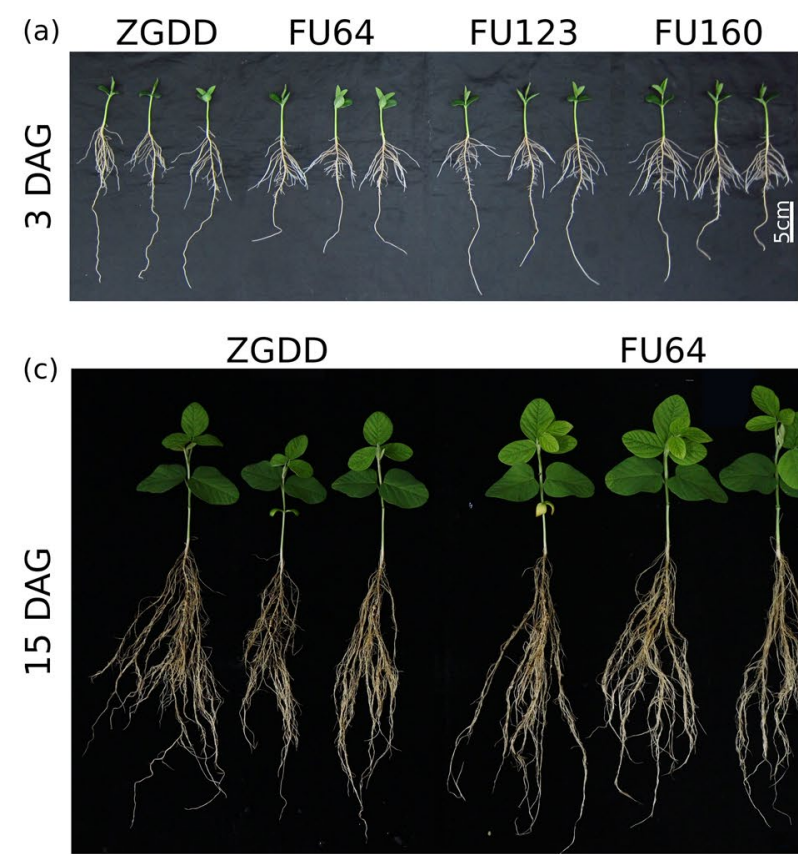

(d)

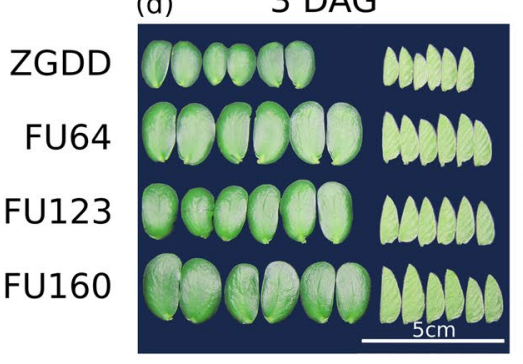

FU64 (e)

7 DAG

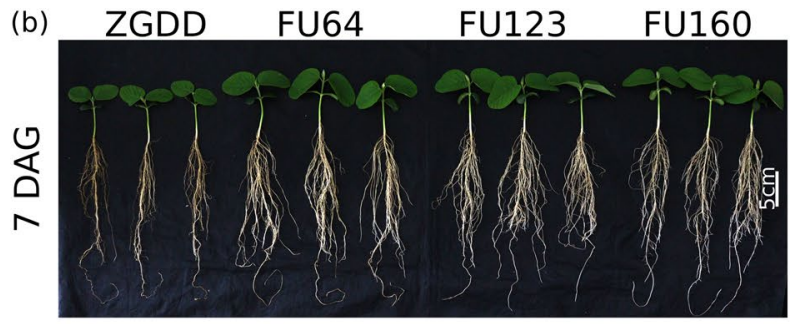

FU123

FU160
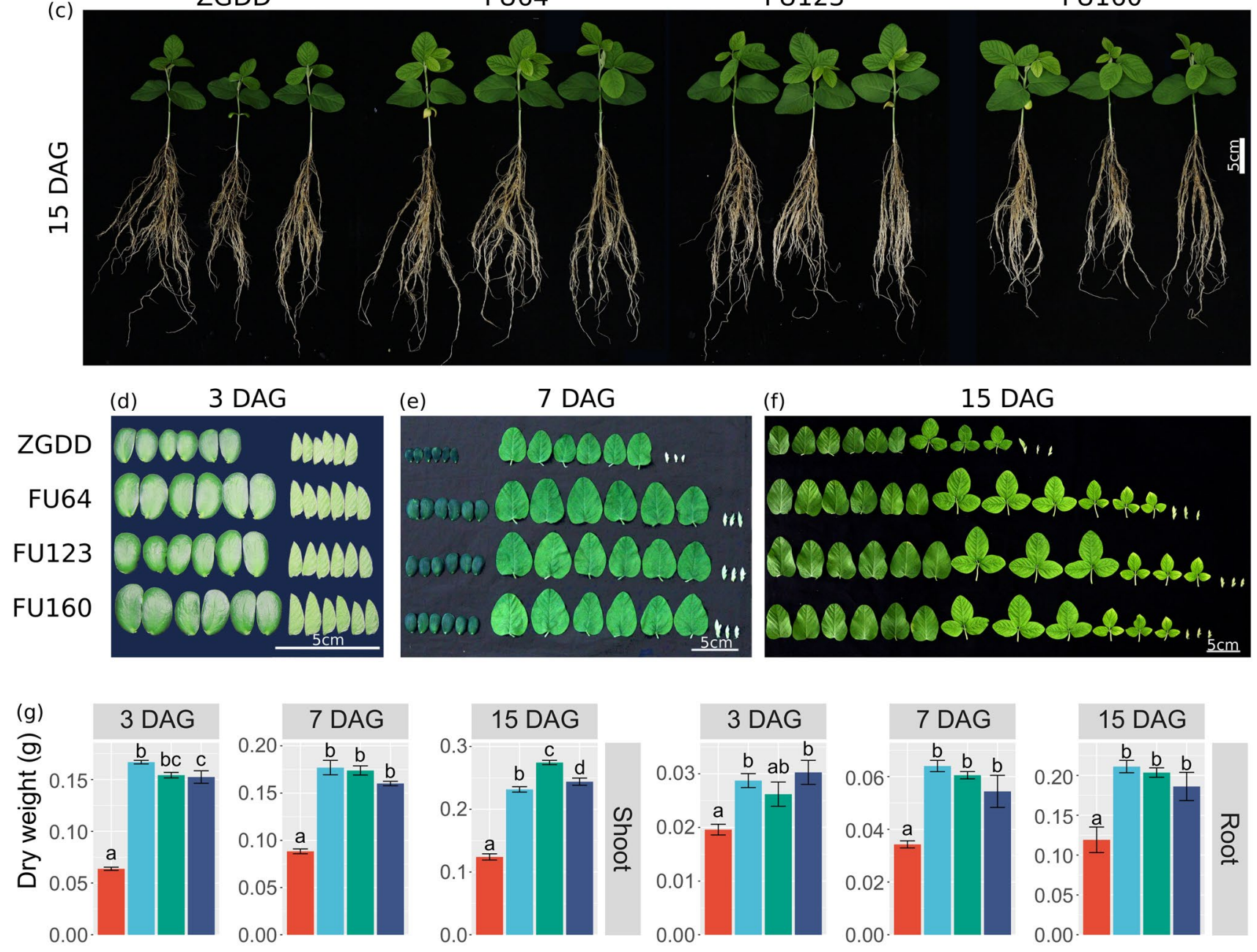

ZGDD

FU64

FU123

FU160

Fig. 2 Overexpression of GmFULa enhances biomass accumulation. a Transgenic plants overexpressing GmFULa reach the same growth stage as control soybean ZGDD at 3 days after germination (DAG). b Transgenic plants overexpressing GmFULa have bigger unifoliolate leaves than control soybean ZGDD at 7 DAG. c Transgenic plants overexpressing GmFULa have one more trifoliolate leaf than control soybean ZGDD at 15 DAG. d-f Cotyledons and leaves of transgenic plants overexpressing GmFULa and control soybean ZGDD

fully expanded trifoliolate leaves of three plants in the V2 stage and weighed. The content of chlorophyll of overexpression plants were significantly higher than those of the wild-type control (Fig. 5a). Correspondingly, the leaf-level at 3 (d), 7 (e), and 15 (f) DAG. g Overexpression of GmFULa promotes the accumulation of dry biomass at 3,7 and 15 DAG $(n=5)$. ZGDD: control soybean Zigongdongdou (transgenic receptor). FU64, FU123 and FU160 are independent transgenic lines overexpressing GmFULa. Bar indicates $5 \mathrm{~cm}$. The data represent the mean \pm SE of five biological replicates with letters showing if there is a significant difference between groups (One-Way ANOVA; Tukey HSD test at, $<0.05$ )

photosynthesis rates of transgenic plants were also significantly enhanced in the field conditions (Fig. 5b). The content of soluble sugar in leaves of V2 stage and seeds of R6 stage were further detected. Compared to WT, transgenic 
(a)
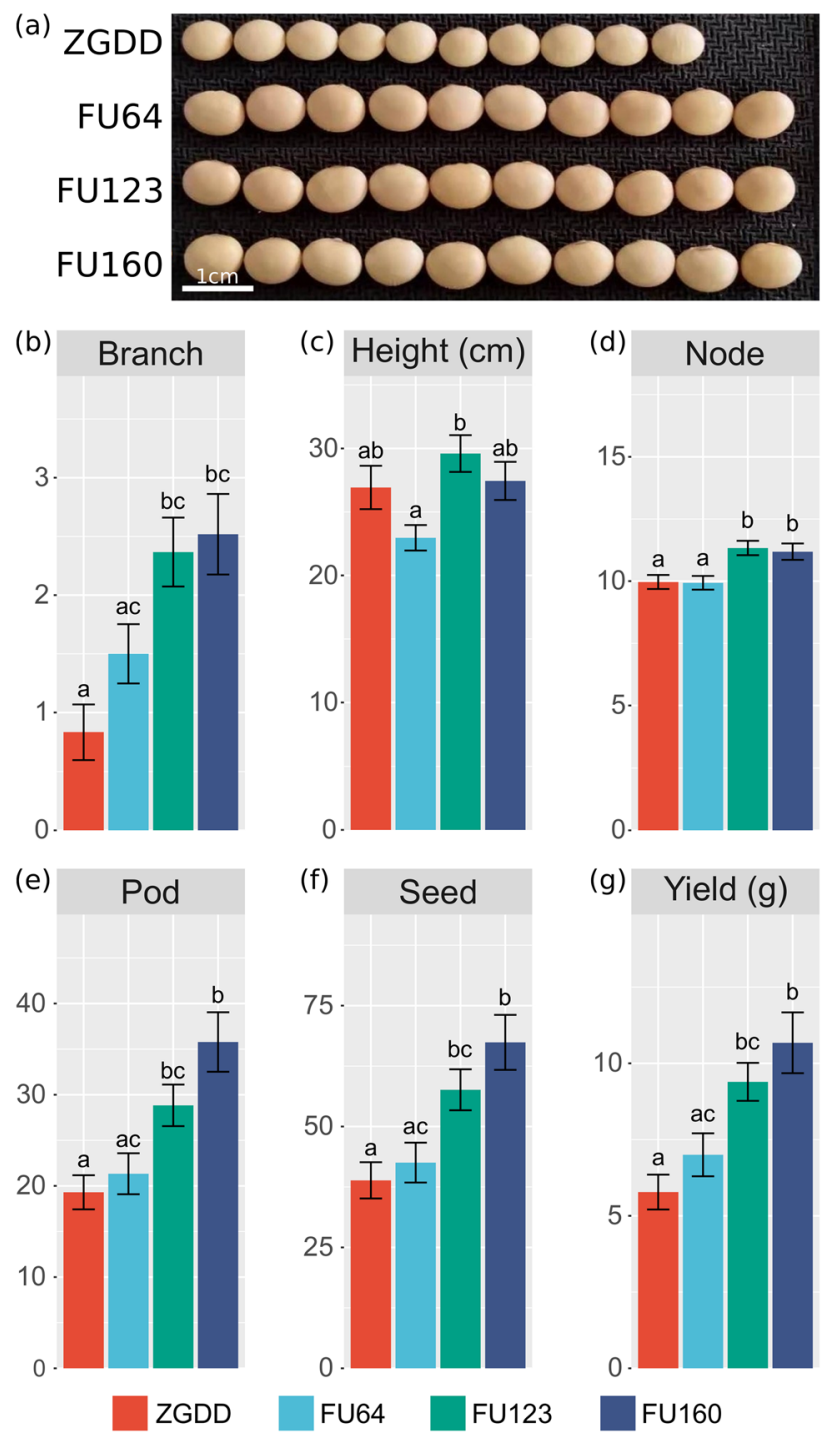

Fig. 3 Overexpression of GmFULa promotes soybean yield. a Representative seed sizes of transgenic lines and control. b-g Yield-related traits branch number (b), plant height $(\mathbf{c})$, node number $(\mathbf{d})$, pod number (e), and seed number (f) and overall yield (g) of transgenic lines and control grown under natural field conditions. The plants were grown in a field in Sanya, Hainan province, China. The data represent the mean $\pm \mathrm{SE}$ from three replicates (ten plants per replicate) with letters showing if there is a significant difference between groups (OneWay ANOVA; Tukey HSD test at, <0.05). ZGDD: control soybean Zigongdongdou (transgenic receptor). FU64, FU123 and FU160 are independent transgenic lines overexpressing GmFULa

overexpression lines exhibited significantly higher levels of whole-soluble sugar both in leaves and seeds (Fig. 6a, b). Consistently, the activity of sucrose synthase of transgenic overexpression lines was higher than that of the wild-type control, though this difference was only significant for FU160 (Fig. 6c). These physiological data indicated that GmFULa enhances assimilation in soybean.

\section{GmFULa binds to the conserved CArG boxes present in the promoter regions of GmSUS12 and GmSUT5}

To understand how GmFULa regulates agronomic and physiologic traits, we further analyzed its protein subcellular localization. By transient expression of GmFULa-GFP (green fluorescent protein) driven by CaMV35S promoter in soybean protoplasts, we found that the fusion protein was localized in the nucleus of soybean protoplasts based on the observation that the GFP signal was exclusively co-localized with the mCherry-labeled nuclear signal (Fig. 6d). These results were in line with the prediction that GmFULa should be a transcription factor.

Considering that soluble sugar synthesis was promoted in the transgenic lines, it is a reasonable hypothesis that GmSUSs and GmSUTs should be regulated by GmFULa. To clarify this hypothesis, we first performed a qPCR experiment to compare the expression of $12 \mathrm{GmSUSs}$ and eight GmSUTs, and found that most GmSUSs and GmSUTs (especially GmSUS12 and GmSUT5) had significantly higher expression in overexpression lines FU64, FU123 and FU160 leaves of V3 stage than in control plant ZGDD. This result suggests that GmSUS and GmSUT are regulated by GmFULa in soybean growth (Fig. 6e).

To further confirm whether GmFULa regulates GmSUS and GmSUT directly, GmSUS12 and GmSUT5 were selected for further analysis. In the 2,000 bp upstream promoter region of both GmSUS12 and GmSUT5, we found three (SA-SC) and seven (TA-TG) FUL-combining CArG boxes, respectively (Fig. 6f), which indicated that GmFULa might combine these boxes to regulate the expression of GmSUS12 and GmSUT5. Then we performed an EMSA, and found a shift for all detected CArG boxes (SA-SC and TA-TG) as predicted, confirming that GmFULa can physically bind to the promoters of GmSUS12 and GmSUT5 (Fig. 6f). Furthermore, a yeast one hybrid experiment also showed that GmFULa binds to GmSUS12 promoter. These results indicate that GmFULa promotes the activity of sucrose synthesis and transport-related genes in soybean.

\section{Discussion}

FRUITFULL (FUL), a MADS-box transcription factor, is essential in the network that regulates the initiation of shoots and buds, the transformation of reproductive growth and the development of organs. In Arabidopsis thaliana, FUL down-regulates AP2 (APETALA2) and IND (INDE$H I S C E N T$ ) and promotes pod elongation (Di Marzo et al. 2020). In cucumber, CsFUL1 regulates fruit length (Zhao et al. 2019). DEFH28 from Antirrhinum majus regulates carpel wall differentiation and fruit ripening (Müller et al. 2001). Bemer et al. (2012) found that TDR4/FUL1 and 


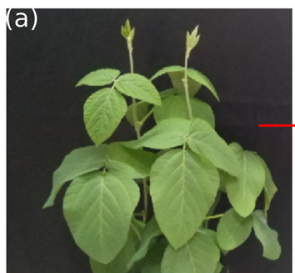

(c)
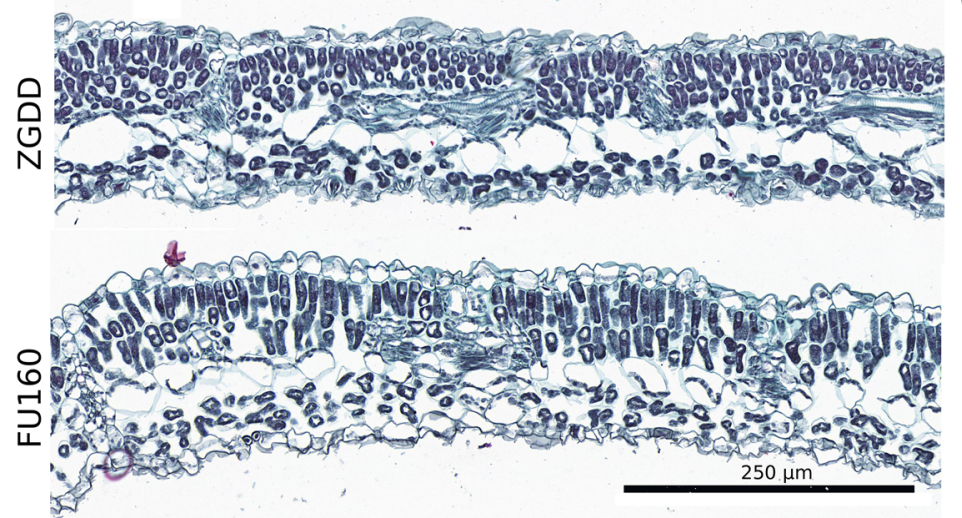

(e)

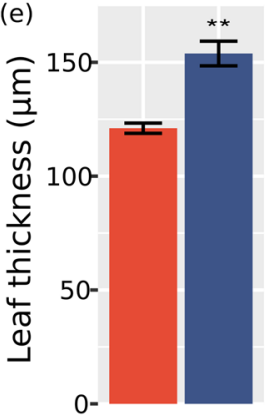

(g) 6-

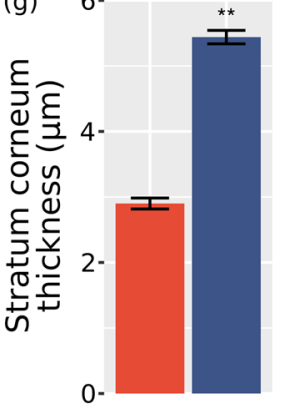

ZGDD

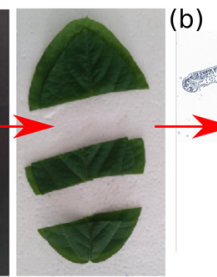

(b)
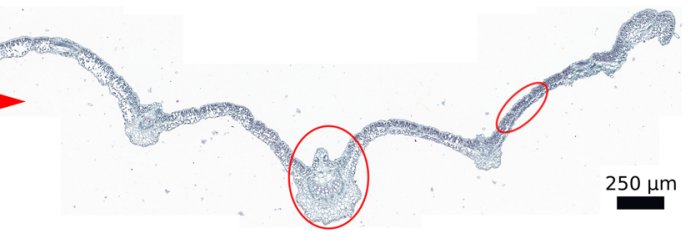

(d)

(f)

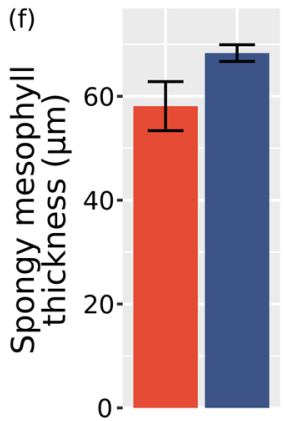

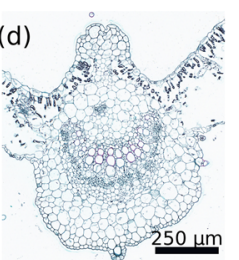
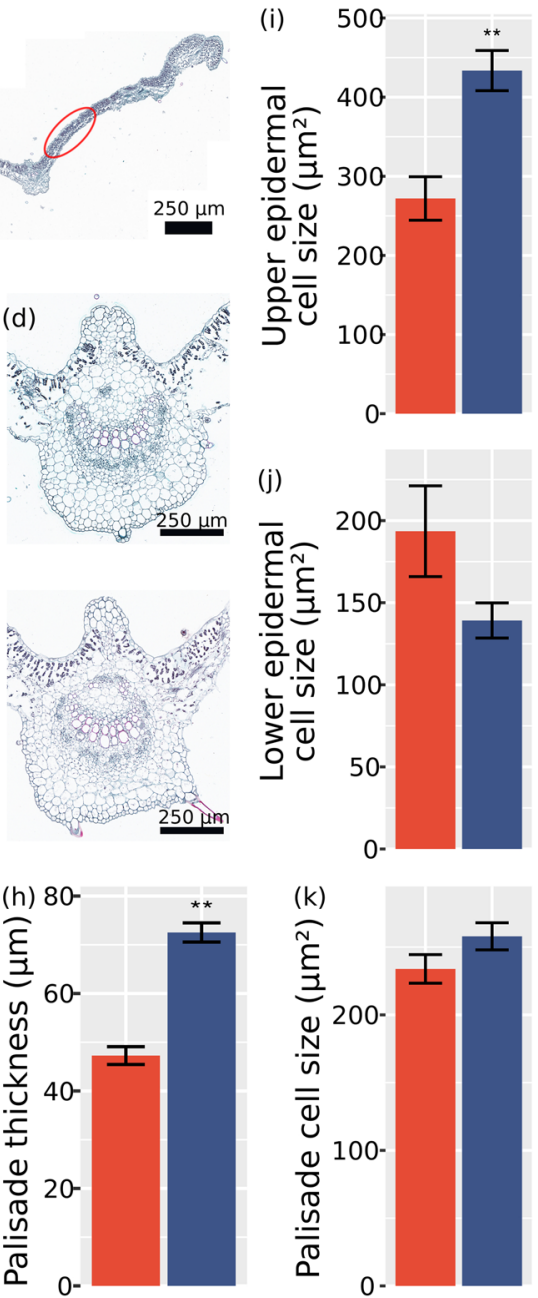

Fig. 4 Overexpression of GmFULa increases the number and size of leaves cell. a, b Leaf sampling method for cell morphology analysis. c Cell morphology of mesophyll of cross section in wild-type and transgenic plants. d Cell morphology of leaf vein cross section in wild-type and transgenic plants. $\mathbf{e}-\mathbf{k}$ Comparison of cell morphology

MBP7/FUL2 affected fruit ripening independent of ethylene. Similarly, our previous study found that GmFULa is specifically expressed in flowers and pods, and is related to photo-thermal adaptation of soybean (Jia et al. 2015). However, we knew less about the exact role of GmFULa on soybean maturity and yield and how it works.

Using publicly available whole-genome resequencing data, we found that GmFULa had two major haplotypes, GmFULa-HO1 and GmFULa-HO2. The proportion of GmFULa-HO2 is highest in cultivated soybeans from Middle and South China and second highest in cultivated soybeans from Northeast China, but nearly absent in wild soybeans. These results indicate that GmFULa-HO2 is an elite allele to be highly selected during domestication and improvement in different tissues. ZGDD: control soybean Zigongdongdou (transgenic receptor). FU160 is the typical transgenic line overexpressing GmFULa. The data represent mean \pm SE from three replicates. $* * p<0.01$ (Student's $t$-test). Scale bars are $250 \mu \mathrm{m}$

of cultivated soybeans and might promote the expansion of soybeans from high latitudes to low latitudes.

Different from our expectation that GmFULa might regulate soybean maturity and improve soybean adaptation, GmFULa overexpression does not alter the maturity structure, or the durations of vegetative and reproductive growth, which is similar with FUL (AGL8) of Arabidopsis and AaFUL1 of Anthurium (Gu et al. 1998; Ma et al. 2019). However, the $F U L$ homologs in Medicago truncatula and Platanus acerifolia promote flowering (Jaudal et al. 2015; Zhang et al. 2019a). In combination with the observation that GmFULa promotes vegetative growth, GmFUL might function in a novel mechanism, as its ancestral homolog Dt2 does in determining semi-determinacy (Liu et al. 2016). 


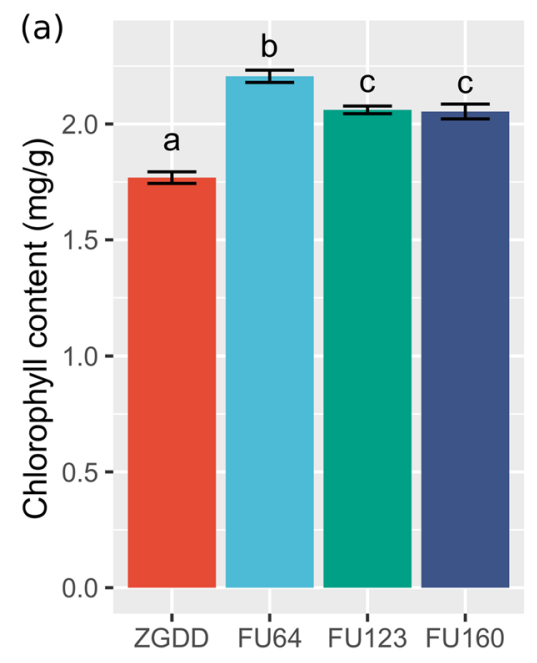

Fig. 5 Overexpression of GmFULa increases the content of chlorophyll and promotes the rate of photosynthesis. a Chlorophyll content in control and transgenic plant leaves. The data represent mean $\pm \mathrm{SE}$ from three biological replicates with letters showing if there is a significant difference between groups (One-Way ANOVA; Tukey HSD test at, <0.05). b Photosynthesis in control and transgenic plants.

GmFULa has an important role in plant architecture through regulating the branch number, node number, leaf size, and leaf thickness. However, GmFULa had no significant effect on plant height when overexpressed. Moreover, it promoted the dry mass accumulation of both root and shoot and ultimately increased yield. In contrast, GmAPla was found to control flowering time and plant height (Chen et al. 2020). These results indicate that GmFULa has pleiotropic roles on soybean growth and development. GmFULa promotes soybean yield, which increases adaptation. It is significantly different from known maturity loci, which regulate soybean maturity to improve soybean adaptation.

GmFULa regulates the source-sink balance. Before reproduction, GmFULa promotes vegetative growth and increases biomass accumulation, as indicated by the observations that chlorophyll content, photosynthesis rate, sucrose synthase activity, soluble sugar content, node number and branch number were all increased in transgenic soybeans compared to the wild-type soybean. However, considering that the number and size of seeds and the final yield were increased in transgenic soybeans compared to the wild-type soybean, GmFULa effectively promotes the source-sink transition during reproduction. Although the photosynthesis rates were smaller than the ones in Koester et al. (2016), our results were consistent with Lin et al. (2015) for the wildtype soybean Zigongdongdou. It might be possibly due to the genetic background difference: different from the soybeans in Koester et al. (2016) which are all maturity group III cultivars, Zigongdongdou is a MGX cultivar. Moreover, both the chlorophyll content and the photosynthesis rate

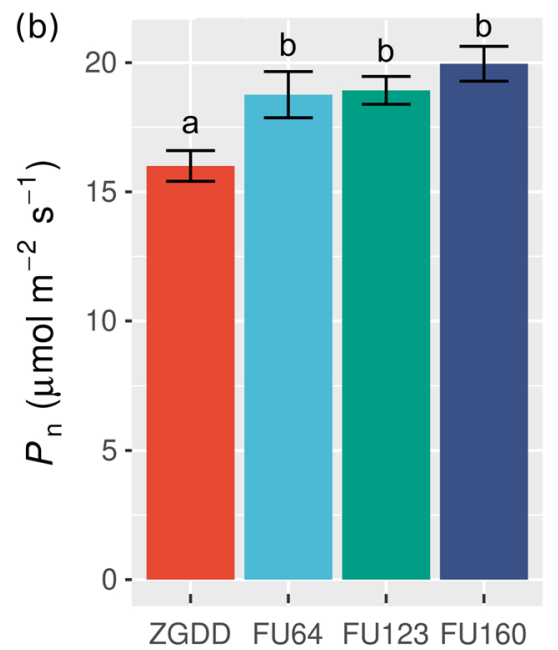

ZGDD: control soybean Zigongdongdou transgenic receptor. FU64, FU123 and FU160 are the independent transgenic lines overexpressing GmFULa. The data represent mean $\pm \mathrm{SE}$ from fifteen biological replicates with letters showing if there is a significant difference between groups (One-Way ANOVA; Tukey HSD test at, <0.05)

were improved, which is consistent with the observation that photosynthesis rate is highly correlated with chlorophyll contents (Buttery and Buzzell 1977). Considering that the enhancement of leaf-level photosynthesis benefited from $\mathrm{chl}$ mutant did not necessarily resulted in canopy-level improvement (Slattery et al. 2016, 2017), it is necessary to further explore the effects of GmFULa on canopy-level processes.

More importantly, consistent with our observation that GmFULa regulates the source-sink balance, it also regulates the expression of the sucrose synthase GmSUS12 and the sucrose transporter GmSUT5 through binding to their promoters. SUS is a key enzyme of sucrose metabolism, with an important role in the process of yield formation (Gessler 2021). Overexpressing potato SUS gene can increase cotton yield significantly (Xu et al. 2012). Moreover, it is also reported to be related to caryopsis development in rice, nitrogen fixation in legumes and plant response to stresses (Huang et al. 1996; Arrese-Igor et al. 1999; Xiao et al. 2014). SUTs are a kind of typical membrane binding protein, which are widely distributed in various tissues and organs of higher plants (Barker et al. 2000; Williams et al. 2000). They are responsible for the transmembrane transport of sucrose, and have a significant role in sucrose entering and leaving phloem, sucrose storage and sucrose supply to the sink tissues (Breia et al. 2020; Wang et al. 2020). Combining the increase in soluble sugar in leaves and seeds in transgenic lines, a working model of GmFULa was proposed (Fig. 7). In the model, GmFULa regulates the expression of SUS to promote sucrose biosynthesis in the source organ leaf. GmFULa also regulates the expression of SUT 

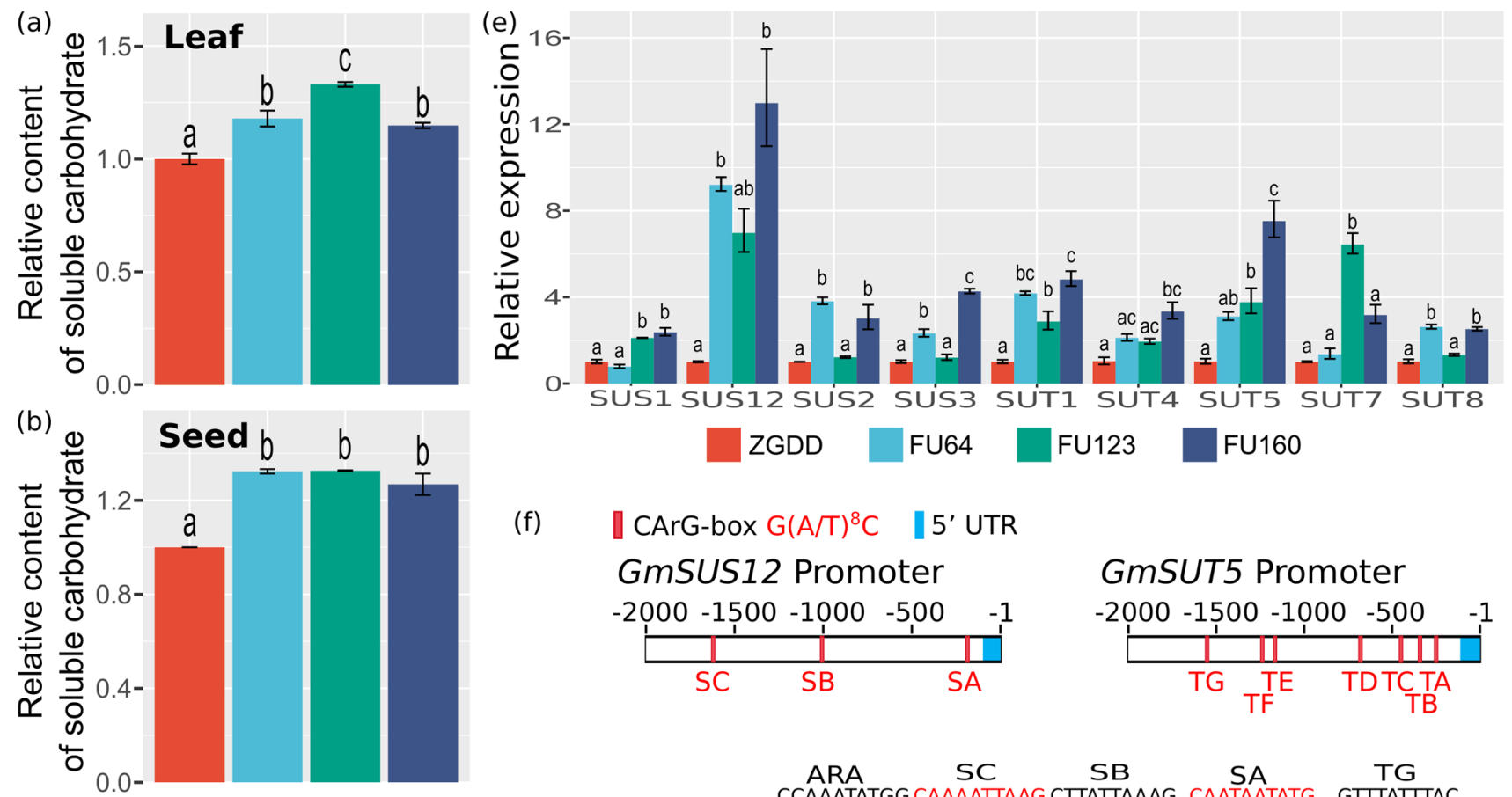

\CArG-box G(A/T $)^{8} \mathrm{C} \quad$ | 5' UTR
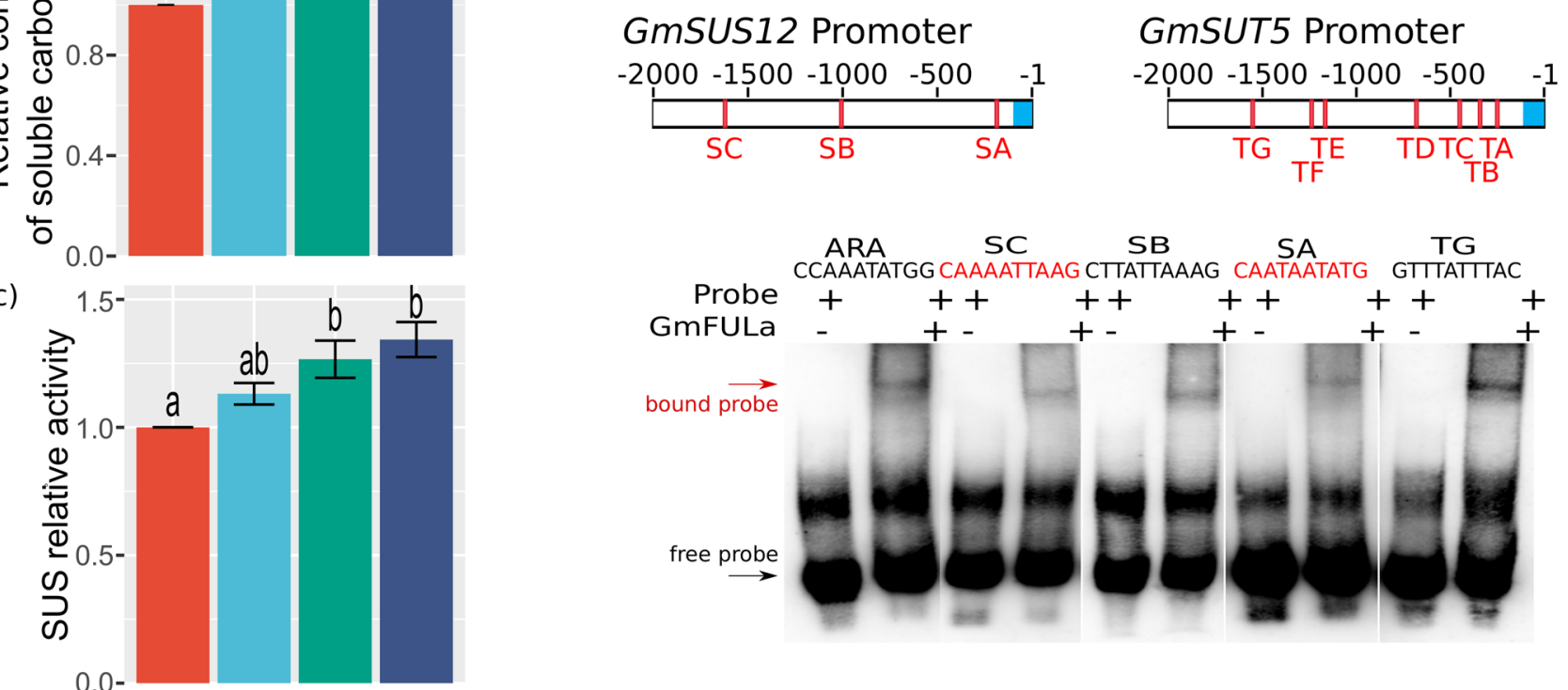

(d)
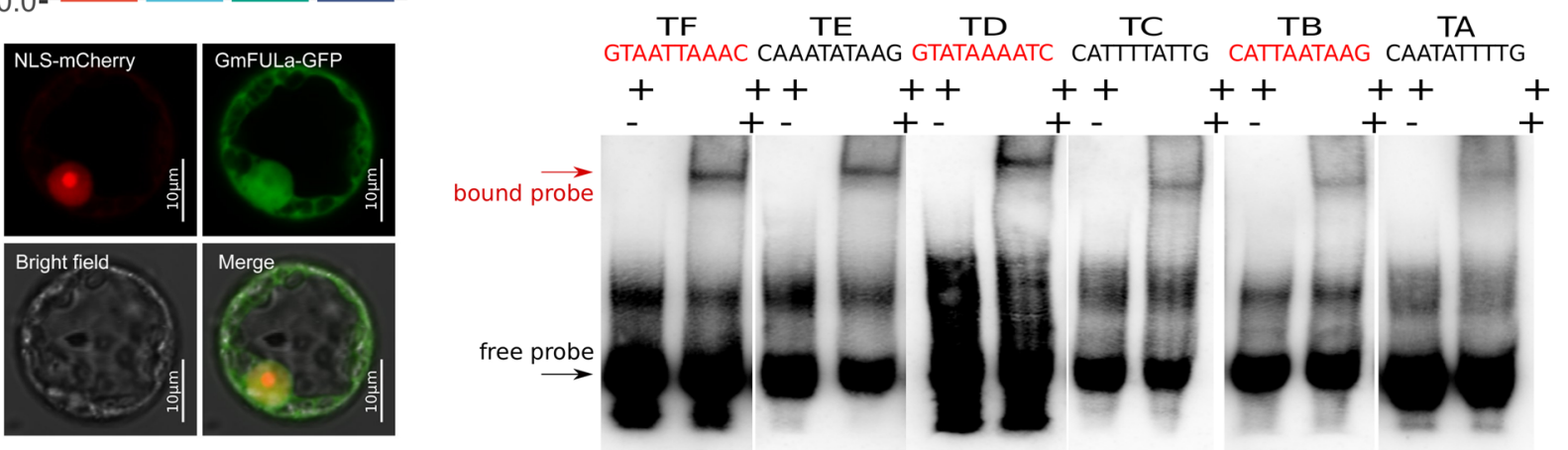

Fig. 6 GmFULa regulates sucrose synthases and transporters to increase soluble sugar content of soybean leaves and seeds. a Relative content of soluble carbohydrate in leaves of transgenic lines and control plant. b Relative content of soluble carbohydrate in seeds of transgenic lines and control plant. c The activity of sucrose synthase in leaves of transgenic lines and control plant. d Subcellular localization of GmFULa in soybean protoplasts. GFP and GmFULa-GFP fusions under the control of the CaMV35S promoter were transiently expressed in soybean protoplasts. Bar $=10 \mu \mathrm{m}$. e Relative expression levels of GmSUSs and GmSUTs in transgenic lines and the control. Soybean GmActin was used as an internal control. a-c, e The data represent mean $\pm \mathrm{SE}$ from three biological replicates with letters showing if there is a significant difference between groups (OneWay ANOVA; Tukey HSD test at, <0.05). f GmFULa binds to the GmSUS12 and GmSUT5 promoters. Schematic diagram of the 2,000 bp GmSUS12 and GmSUT5 promoter regions showed three and seven CArG boxes, respectively. EMSA assay testing the binding of GmFULa to the GmSUS12 and GmSUT5 promoter fragments. Two 40 bp single-strand oligonucleotide probes containing CArG box motif with 16 bp flanking sequences were synthesized and labeled with biotin. + and - indicate the presence and absence of the corresponding probe or protein. ZGDD: control soybean Zigongdongdou (transgenic receptor). FU64, FU123 and FU160 are the independent transgenic lines overexpressing GmFULa 
Fig. 7 Working model for regulation of soybean vegetative growth, cell development, and yield by GmFULa. GmFULa regulates both the sucrose synthases (SUS) and the sucrose transporters to synchronize the sucrose biosynthesis (energy generation and assimilation) in the source organ (leaf) and the sucrose transportation (energy transportation) to the sink organ (pod) to finally promote yield

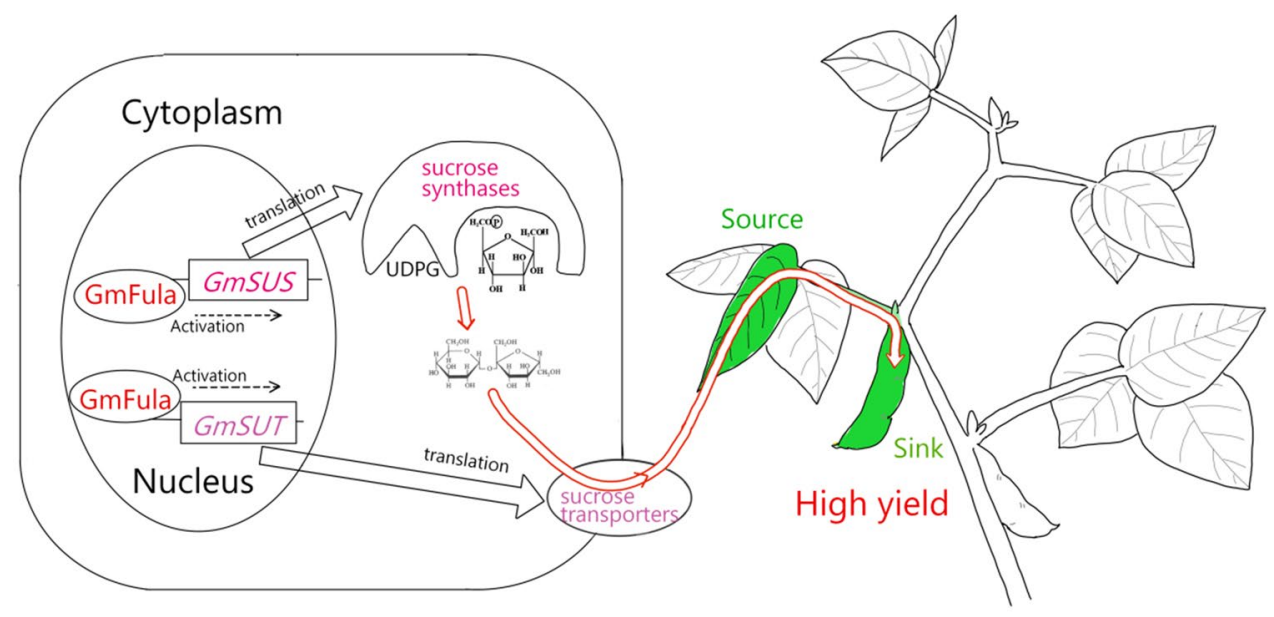

to promote sucrose transportation into the sink organ pod. Thus, GmFULa can synchronize both sucrose biosynthesis and transportation to increase the source-sink transition rate of the photosynthesis product and the photosynthesis rate is promoted as a result, which is consistent with the hypothesis proposed by Ainsworth et al. (2004). Moreover, as indicated by the observation that FUL homologs promote pod development, GmFULa efficiently promotes the utilization of sucrose in the sink organ pod to increase the final yield suggesting that GmFULa might also function in pod development. Moreover, GmFULa promotes root development, which means that more nutrients can be absorbed to support development of the above ground parts of plants.

GmFULa provides a new option for yield improvement of soybean. Soybean is a short-day crop that is sensitive to photoperiod, and the yield is highly dependent on adaption to local photo-thermal environments (Yue et al. 2017). Thus, in the breeding history of soybean, the first trait to address is photoperiod sensitivity, specifically, modifying maturity to match the soybean photoperiod sensitivity to local environments. Multiple soybean maturity loci have been identified. With the discovery and application of maturity loci, soybean has expanded to higher and lower latitudes, resulting in huge increases in soybean production. If the production increase mainly resulting from maturity adaption can be called the first-generation revolution of soybean breeding, the second-generation revolution of soybean breeding will be the improvement of traits other than maturity. In current breeding programs/strategies, the parental lines with different genetic backgrounds of maturity loci are challenging in conventional hybrid breeding, requiring efforts to find optimal photo-thermal environments for their filial lines through field experiments in different locations. Many known yield loci are highly linked to maturity traits; thus, transgenic modification of their causal genes might also have secondary effects that alter the maturity trait, and as a consequence, the main effect of yield will be uncertain if the optimal photo-thermal environments are changed.
However, because GmFULa can improve the yield without altering the maturity, it is possible to directly improve elite cultivars without changing maturity. For an elite cultivar, we can modify the expression of GmFULa through transgenic overexpression or through gene editing to introduce an enhancer element, remove an inhibitor element or make a haplotype shift, consequently enhancing the yield capacity without significantly changing the optimal ecoregion.

In summary, we have functionally characterized GmFULa, a member of the MADS-box family in soybean. GmFULa has pleiotropic roles in soybean growth and development. It increases soybean adaptation through promoting vegetative growth and reproductive growth to increase soybean yield. It promotes source accumulation and the sink transformation, but does not affect maturity. Overexpression of GmFULa, thus, provides a new way to increase soybean yield and soybean adaptation.

Supplementary Information The online version contains supplementary material available at https://doi.org/10.1007/s00299-021-02752-y.

Acknowledgements We thank Mrs Jinlu Tao, Mr Haifeng Hong and Mr Enoch Sapey from the Institute of Crop Sciences, Chinese Academy of Agricultural Sciences for their assistance in soybean planting and management. This work was supported by the National Natural Science Foundation of China (32001573), the National Key R\&D Program of China (2017YFD0101400), the China Agriculture Research System (CARS-04) and the CAAS Agricultural Science and Technology Innovation Project.

Author contribution statement $\mathrm{YY}, \mathrm{BJ}, \mathrm{TH}$, and TL conceived the project; YY, BJ and SS performed the field experiments. YY, TL, BJ, SS, JL, HW, and BS performed the indoor experiments; BJ, and YY performed the data analysis. YY, BJ, TH, TL, and HY wrote the manuscript.

\section{Declarations}

Conflict of interest The authors declare no conflict of interests.

Ethical approval This article does not contain any studies with human participants or animals performed by any of the authors. 
Open Access This article is licensed under a Creative Commons Attribution 4.0 International License, which permits use, sharing, adaptation, distribution and reproduction in any medium or format, as long as you give appropriate credit to the original author(s) and the source, provide a link to the Creative Commons licence, and indicate if changes were made. The images or other third party material in this article are included in the article's Creative Commons licence, unless indicated otherwise in a credit line to the material. If material is not included in the article's Creative Commons licence and your intended use is not permitted by statutory regulation or exceeds the permitted use, you will need to obtain permission directly from the copyright holder. To view a copy of this licence, visit http://creativecommons.org/licenses/by/4.0/.

\section{References}

Ainsworth EA, Rogers A, Nelson R, Long SP (2004) Testing the "source-sink" hypothesis of down-regulation of photosynthesis in elevated $\left[\mathrm{CO}_{2}\right]$ in the field with single gene substitutions in Glycine max. Agric for Meteorol 122:85-94

Alvarez-Buylla ER, García-Ponce B, MdlP S, Espinosa-Soto C, GarcíaGómez ML, Piñeyro-Nelson A, Garay-Arroyo A (2019) MADSbox genes underground becoming mainstream: plant root developmental mechanisms. New Phytol 223:1143-1158

Arrese-Igor C, Gonzalez E, Gordon A, Minchin F, Galvez L, Royuela M, Cabrerizo P, Aparicio-Tejo P (1999) Sucrose synthase and nodule nitrogen fixation under drought and environmental stresses. Symbiosis 27:189-212

Balanza V, Martinez-Fernandez I, Sato S, Yanofsky MF, Ferrandiz C (2019) Inflorescence meristem fate is dependent on seed development and FRUITFULL in Arabidopsis thaliana. Front Plant Sci 10:1622

Barker L, Kühn C, Weise A, Schulz A, Gebhardt C, Hirner B, Hellmann H, Schulze W, Ward JM, Frommer WB (2000) SUT2, a putative sucrose sensor in sieve elements. Plant Cell 12:1153-1164

Bemer M, Karlova R, Ballester AR, Tikunov YM, Bovy AG, WoltersArts M, Rossetto PdB, Angenent GC, de Maagd RA (2012) The tomato FRUITFULL homologs TDR4/FUL1 and MBP7/FUL2 regulate ethylene-independent aspects of fruit ripening. Plant Cell 24:4437-4451

Bemer M, van Mourik H, Muino JM, Ferrandiz C, Kaufmann K, Angenent GC (2017) FRUITFULL controls SAUR10 expression and regulates Arabidopsis growth and architecture. J Exp Bot 68:3391-3403

Berbel A, Ferrándiz C, Hecht V, Dalmais M, Lund OS, Sussmilch FC, Taylor SA, Bendahmane A, Ellis THN, Beltrán JP, Weller JL, Madueño F (2012) VEGETATIVE1 is essential for development of the compound inflorescence in pea. Nat Commun 3:797

Bianchi JS, Quijano A, Gosparini CO, Morandi EN (2020) Changes in leaflet shape and seeds per pod modify crop growth parameters, canopy light environment, and yield components in soybean. Crop J 8:351-364

Breia R, Conde A, Conde C, Fortes AM, Granell A, Gerós H (2020) VvERD6113 is a grapevine sucrose transporter highly up-regulated in response to infection by Botrytis cinerea and Erysiphe necator. Plant Physiol Bioch 154:508-516

Busch FA (2018) Photosynthetic gas exchange in land plants at the leaf level. In: Covshoff S (ed) Photosynthesis: methods and protocols. Springer, New York, pp 25-44

Buttery BR, Buzzell RI (1977) The relationship between chlorophyll content and rate of photosynthesis in soybeans. Can J Plant Sci $57: 1-5$
Chen L, Nan H, Kong L, Yue L, Yang H, Zhao Q, Fang C, Li H, Cheng Q, Lu S, Kong F, Liu B, Dong L (2020) Soybean APl homologs control flowering time and plant height. J Integr Plant Biol 62:1868-1879

Di Marzo M, Herrera-Ubaldo H, Caporali E, Novak O, Strnad M, Balanza V, Ezquer I, Mendes MA, de Folter S, Colombo L (2020) SEEDSTICK controls Arabidopsis fruit size by regulating cytokinin levels and FRUITFULL. Cell Rep 30:2846-2857

Ferrandiz C, Liljegren SJ, Yanofsky MF (2000) Negative regulation of the SHATTERPROOF genes by FRUITFULL during Arabidopsis fruit development. Science 289:436-438

Frearson EM, Power JB, Cocking EC (1973) The isolation, culture and regeneration of Petunia leaf protoplasts. Dev Biol 33:130-137

Gessler A (2021) Sucrose synthase - an enzyme with a central role in the source-sink coordination and carbon flow in trees. New Phytol 229:8-10

Gu Q, Ferrandiz C, Yanofsky MF, Martienssen R (1998) The FRUITFULL MADS-box gene mediates cell differentiation during Arabidopsis fruit development. Development 125:1509-1517

Hu D, Zhang H, Du Q, Hu Z, Yang Z, Li X, Wang J, Huang F, Yu D, Wang H, Kan G (2020) Genetic dissection of yield-related traits via genome-wide association analysis across multiple environments in wild soybean (Glycine soja Sieb. and Zucc.). Planta 251:39

Huang JW, Chen JT, Yu WP, Shyur LF, Wang AY, Sung HY, Lee PD, $\mathrm{Su}$ JC (1996) Complete structures of three rice sucrose synthase isogenes and differential regulation of their expressions. Biosci Biotechnol Biochem 60:233-239

Huang Y, Wang L, Hu S, Luo X, Cao Y (2020) Overexpression of the bamboo sucrose synthase gene (BeSUS5) improves cellulose production, cell wall thickness and fiber quality in transgenic poplar. Tree Genet Genomes 16:75

Jaakola L, Poole M, Jones MO, Kämäräinen-Karppinen T, Koskimäki JJ, Hohtola A, Häggman H, Fraser PD, Manning K, King GJ, Thomson H, Seymour GB (2010) A SQUAMOSA MADS box gene involved in the regulation of anthocyanin accumulation in bilberry fruits. Plant Physiol 153:1619-1629

Jaudal M, Zhang L, Che C, Putterill J (2015) Three Medicago MtFUL genes have distinct and overlapping expression patterns during vegetative and reproductive development and 35S:MtFULb accelerates flowering and causes a terminal flower phenotype in Arabidopsis. Front Genet 6:50

Jeong N, Suh SJ, Kim M-H, Lee S, Moon J-K, Kim HS, Jeong S-C (2012) $L n$ is a key regulator of leaflet shape and number of seeds per pod in soybean. Plant Cell 24:4807-4818

Jia Z, Jiang B, Gao X, Yue Y, Fei Z, Sun H, Wu C, Sun S, Hou W, Han T (2015) GmFULa, a FRUITFULL homolog, functions in the flowering and maturation of soybean. Plant Cell Rep 34:121-132

Jiang B, Zhang S, Song W, Khan MAA, Sun S, Zhang C, Wu T, Wu C, Han T (2019) Natural variations of FT family genes in soybean varieties covering a wide range of maturity groups. BMC Genomics 20:230

Koester RP, Nohl BM, Diers BW, Ainsworth EA (2016) Has photosynthetic capacity increased with 80 years of soybean breeding? An examination of historical soybean cultivars. Plant Cell Environ 39:1058-1067

Lam H-M, Xu X, Liu X, Chen W, Yang G, Wong F-L, Li M-W, He W, Qin N, Wang B, Li J, Jian M, Wang J, Shao G, Wang J, Sun SS-M, Zhang G (2010) Resequencing of 31 wild and cultivated soybean genomes identifies patterns of genetic diversity and selection. Nat Genet 42:1053-1059

Langdale JA, Lane B, Freeling M, Nelson T (1989) Cell lineage analysis of maize bundle sheath and mesophyll cells. Dev Biol 133:128-139 
Li S, Chen K, Grierson D (2019) A critical evaluation of the role of ethylene and MADS transcription factors in the network controlling fleshy fruit ripening. New Phytol 221:1724-1741

Lin K, Liu X, Sun S, Chen L, Han T, Hou W (2015) Salt tolerance analysis of TaNHX2 over-expression transgenic soybean. Sci Agric Sin 48:3998-4007

Liu Y, Zhang D, Ping J, Li S, Chen Z, Ma J (2016) Innovation of a regulatory mechanism modulating semi-determinate stem growth through artificial selection in soybean. PLoS Genet 12:e1005818

Ma G, Zou Q, Shi X, Tian D, Sheng Q (2019) Ectopic expression of the AaFUL1 gene identified in Anthurium andraeanum affected floral organ development and seed fertility in tobacco. Gene 696:197-205

Maheepala DC, Emerling CA, Rajewski A, Macon J, Strahl M, PabonMora N, Litt A (2019) Evolution and diversification of FRUITFULL genes in Solanaceae. Front Plant Sci 10:43

Mandel MA, Yanofsky MF (1995) The Arabidopsis AGL8 MADS box gene is expressed in inflorescence meristems and is negatively regulated by APETALA1. Plant Cell 7:1763-1771

Müller BM, Saedler H, Zachgo S (2001) The MADS-box gene DEFH28 from Antirrhinum is involved in the regulation of floral meristem identity and fruit development. Plant J 28:169-179

Nguyen CX, Paddock KJ, Zhang Z, Stacey MG (2021) GmKIX8-1 regulates organ size in soybean and is the causative gene for the major seed weight QTL $q S w 17-1$. New Phytol 229:920-934

Paz MM, Shou H, Guo Z, Zhang Z, Banerjee AK, Wang K (2004) Assessment of conditions affecting Agrobacterium-mediated soybean transformation using the cotyledonary node explant. Euphytica 136:167-179

Sayama T, Tanabata T, Saruta M, Yamada T, Anai T, Kaga A, Ishimoto M (2017) Confirmation of the pleiotropic control of leaflet shape and number of seeds per pod by the $L n$ gene in induced soybean mutants. Breed Sci 67:363-369

Sehra B, Franks RG (2017) Redundant CArG box cis-motif activity mediates SHATTERPROOF2 transcriptional regulation during Arabidopsis thaliana gynoecium development. Front Plant Sci $8: 1712$

Singsaas EL, Ort DR, Delucia EH (2004) Elevated $\mathrm{CO}_{2}$ effects on mesophyll conductance and its consequences for interpreting photosynthetic physiology. Plant Cell Environ 27:41-50

Slattery RA, Grennan AK, Sivaguru M, Sozzani R, Ort DR (2016) Light sheet microscopy reveals more gradual light attenuation in light-green versus dark-green soybean leaves. J Exp Bot 67:4697-4709

Slattery RA, VanLoocke A, Bernacchi CJ, Zhu X-G, Ort DR (2017) Photosynthesis, light use efficiency, and yield of reduced-chlorophyll soybean mutants in field conditions. Front Plant Sci 8:549

Taylor SC, Nadeau K, Abbasi M, Lachance C, Nguyen M, Fenrich J (2019) The ultimate qPCR experiment: producing publication quality, reproducible data the first time. Trends Biotechnol 37:761-774

Van Roekel RJ, Purcell LC, Salmerón M (2015) Physiological and management factors contributing to soybean potential yield. Field Crop Res 182:86-97

Wang S, Lu G, Hou Z, Luo Z, Wang T, Li H, Zhang J, Ye Z (2014) Members of the tomato FRUITFULL MADS-box family regulate style abscission and fruit ripening. J Exp Bot 65:3005-3014

Wang X, Li Y, Zhang H, Sun G, Zhang W, Qiu L (2015) Evolution and association analysis of $G m C Y P 78 A 10$ gene with seed size/weight and pod number in soybean. Mol Biol Rep 42:489-496

Wang D, Liu H, Wang H, Zhang P, Shi C (2020) A novel sucrose transporter gene IbSUT4 involves in plant growth and response to abiotic stress through the ABF-dependent ABA signaling pathway in Sweetpotato. BMC Plant Biol 20:157

Williams LE, Lemoine R, Sauer N (2000) Sugar transporters in higher plants-a diversity of roles and complex regulation. Trends Plant Sci 5:283-290

Xiao X, Tang C, Fang Y, Yang M, Zhou B, Qi J, Zhang Y (2014) Structure and expression profile of the sucrose synthase gene family in the rubber tree: indicative of roles in stress response and sucrose utilization in the laticifers. FEBS J 281:291-305

Xu S-M, Brill E, Llewellyn DJ, Furbank RT, Ruan Y-L (2012) Overexpression of a potato sucrose synthase gene in cotton accelerates leaf expansion, reduces seed abortion, and enhances fiber production. Mol Plant 5:430-441

Xu C, Yin Y, Cai R, Wang P, Ni Y, Guo J, Chen E, Cai T, Cui Z, Liu T, Yang D, Wang Z (2013) Responses of photosynthetic characteristics and antioxidative metabolism in winter wheat to post-anthesis shading. Photosynthetica 51:139-150

Yan L, Hofmann N, Li S, Ferreira ME, Song B, Jiang G, Ren S, Quigley C, Fickus E, Cregan P, Song Q (2017) Identification of QTL with large effect on seed weight in a selective population of soybean with genome-wide association and fixation index analyses. BMC Genom 18:529

Yao T, Park BS, Mao H-Z, Seo JS, Ohama N, Li Y, Yu N, Mustafa NFB, Huang C-H, Chua N-H (2019) Regulation of flowering time by SPL10/MED25 module in Arabidopsis. N Phytol 224:493-504

Yoo S-D, Cho Y-H, Sheen J (2007) Arabidopsis mesophyll protoplasts: a versatile cell system for transient gene expression analysis. Nat Protoc 2:1565-1572

Yue Y, Liu N, Jiang B, Li M, Wang H, Jiang Z, Pan H, Xia Q, Ma Q, Han T, Nian H (2017) A single nucleotide deletion in $J$ encoding GmELF3 confers long juvenility and is associated with adaption of tropic soybean. Mol Plant 10:656-658

Zhang W, Fan S, Pang C, Wei H, Ma J, Song M, Yu S (2013) Molecular cloning and function analysis of two SQUAMOSA-like MADS-box genes from Gossypium hirsutum L. J Integr Plant Biol 55:597-607

Zhang S, Lu S, Yi S, Han H, Zhou Q, Cai F, Bao M, Liu G (2019a) Identification and characterization of FRUITFULL-like genes from Platanus acerifolia, a basal eudicot tree. Plant Sci 280:206-218

Zhang T, Wu T, Wang L, Jiang B, Zhen C, Yuan S, Hou W, Wu C, Han T, Sun S (2019b) A combined linkage and GWAS analysis identifies QTLs linked to soybean seed protein and oil content. Int J Mol Sci 20:5915

Zhao J, Jiang L, Che G, Pan Y, Li Y, Hou Y, Zhao W, Zhong Y, Ding L, Yan S, Sun C, Liu R, Yan L, Wu T, Li X, Weng Y, Zhang X (2019) A functional allele of $C s F U L 1$ regulates fruit length through repressing CSSUP and inhibiting auxin transport in Cucumber. Plant Cell 31:1289-1307

Zhou Z, Jiang Y, Wang Z, Gou Z, Lyu J, Li W, Yu Y, Shu L, Zhao Y, Ma Y, Fang C, Shen Y, Liu T, Li C, Li Q, Wu M, Wang M, Wu Y, Dong Y, Wan W, Wang X, Ding Z, Gao Y, Xiang H, Zhu B, Lee S-H, Wang W, Tian Z (2015) Resequencing 302 wild and cultivated accessions identifies genes related to domestication and improvement in soybean. Nat Biotechnol 33:408-414

Publisher's Note Springer Nature remains neutral with regard to jurisdictional claims in published maps and institutional affiliations. 